\title{
Safety and Efficacy of Semichronic Suprachoroidal Transretinal Stimulation with Femtosecond Laser-induced Porosity and Smooth-surface Electrodes
}

\author{
Yasuo Terasawa, ${ }^{1,2 *}$ Hiroyuki Tashiro, ${ }^{3}$ Yukari Nakano, ${ }^{1,2}$ and Jun Ohta $^{2}$ \\ ${ }^{1}$ Artificial Vision Institute, R \& D Division, Nidek Co., Ltd., 13-2 Hama-cho, Gamagori, Aichi 443-0036, Japan \\ ${ }^{2}$ Materials Science, Nara Institute of Science and Technology, Takayama-cho, Ikoma, Nara 630-0192, Japan \\ ${ }^{3}$ Department of Health Sciences, Kyushu University, 3-1-1 Maidashi, Higashi-ku, Fukuoka 812-8582, Japan
}

(Received June 30, 2017; accepted August 29, 2017)

Keywords: retinal prosthesis, suprachoroidal transretinal stimulation, electrodes

We have been developing a retinal prosthesis based on suprachoroidal transretinal stimulation (STS). From a practical viewpoint, it is essential to confirm the safety and effectiveness of continuous electrical stimulation to the retina. In this study, the electrical stimulation of a rabbit retina for $8 \mathrm{~h}$ a day was conducted for one month, and its safety and efficacy were evaluated by histological, electrophysiological, and electrochemical methods. Results suggested that one month of semichronic electrical stimulation by STS was safe and effective.

\section{Introduction}

In the normal visual system, light from the outside world is projected onto the retina, converted from optical information to chemical and electrical signals, and transmitted to the central nervous system. ${ }^{(1)}$ Losing retinal function causes blindness. However, it has been revealed that some retinal cell layers connecting to the higher region of the visual system remain functional even in a retina that suffers from blindness-causing diseases such as retinitis pigmentosa. ${ }^{(2)}$ A retinal prosthesis sends visual information by artificially stimulating these cell layers. In recent years, many research groups worldwide have developed retinal prostheses, and several types of retinal prostheses have been approved for commercialization. ${ }^{(3,4)}$

Retinal prostheses can be classified into epiretinal, ${ }^{(3)}$ subretinal, ${ }^{(4)}$ and suprachoroidal ${ }^{(5,6)}$ prostheses, depending on where the stimulation electrode array is placed. Our group has been developing a retinal prosthesis based on suprachoroidal transretinal stimulation (STS). In STS, the stimulation electrode array is placed in the sclera. The major advantage of STS is its low invasiveness because there is no need to place the stimulation electrodes in the eye. Furthermore, secure fixation of the stimulation electrode array is possible in STS because the stimulation electrode array is sandwiched by scleral tissue. However, compared with other approaches, the challenge of STS is that a relatively large charge injection is required to induce light sensation ("phosphene") because the distance between the stimulation electrode and the

*Corresponding author: e-mail: yasuo_terasawa@nidek.co.jp

http://dx.doi.org/10.18494/SAM.2018.1652 
retina is large. Little is known about the safety or efficacy of the continuous application of such a large charge injection, particularly in suprachoroidal stimulation. Nayagam et al. showed that continuous chronic electrical stimulation up to $77 \mu \mathrm{C} / \mathrm{cm}^{2}$ was safe by chronically stimulating electrodes implanted in the suprachoroidal spaces of felines for three months. ${ }^{(7)}$ However, in this study, stimulation electrodes were placed between the choroid and the sclera, which is somewhat different from the electrode position in STS (inside the sclera). Furthermore, their stimulation electrodes were made of planar circular platinum; this is also different from the bullet-shaped electrodes used in STS. ${ }^{(8)}$

In this study, one-month semichronic STS was applied to a rabbit retina, and its safety and efficacy were evaluated histologically, electrophysiologically, and electrochemically. Furthermore, to clarify the effectiveness of femtosecond laser-induced porosity (FLiP), ${ }^{(9)}$ which has recently been introduced as a technique to improve the performance of stimulation electrodes, electrical stimulation was applied to both cohorts of smooth-surface electrodes without laser application and FLiP electrodes. A part of this study was presented at a conference. ${ }^{(10)}$

\section{Materials and Methods}

\subsection{Electrode array}

Stimulation electrodes were manufactured by mechanical machining from platinum bulk material. The diameter and height were 0.5 and $0.3 \mathrm{~mm}$, respectively. The stimulation electrodes had a bullet-like hemispherical shape [Fig. 1(e)], and were embedded in the parylene substrate [Figs. 1(a) and 1(e)], and the electrodes and external stimulator were connected using a platinum-iridium wire welded to the stimulation electrode. Two platinum electrodes were embedded in the array. Electrical stimulation was applied to only one of the two electrodes [we refer to this as the "active" electrode: channel 1 in Fig. 1(b)]. The two electrodes were surrounded by dummy electrodes made of parylene. The role of the dummy electrodes was to disperse the mechanical pressure from the electrodes to the choroid and retina. The FLiP electrodes had the porous surface introduced by repeated femtosecond-laser irradiation. The typical diameter and depth of a pore are 10 and $100 \mu \mathrm{m}$ respectively. Both smooth-surface electrodes without laser processing [Fig. 1(c)] and FLiP electrodes [Fig. 1(d)] were used as the stimulation electrode. Active and inactive electrodes had the same surface structures (i.e., if the active electrode had a smooth surface, the inactive electrode also had a smooth surface. If the active electrode had FLiP, the inactive electrode also had FLiP).

\subsection{Implantation}

Seven Japanese white normally-sighted rabbits were used in this study. The animals were anesthetized with inhaled isoflurane. After exposing the sclera by dissecting the conjunctiva and inferior rectus muscle, a scleral pocket approximately $5 \times 5 \mathrm{~mm}^{2}$ was created with a crescent knife at the lower temporal area $9 \mathrm{~mm}$ from the corneal limbus. Then the electrode array was inserted into the scleral pocket, and a cable was sutured onto the sclera. The return electrode, a 0.5-mm-diameter, 3-mm-long platinum bar, was implanted in the upper nasal area 


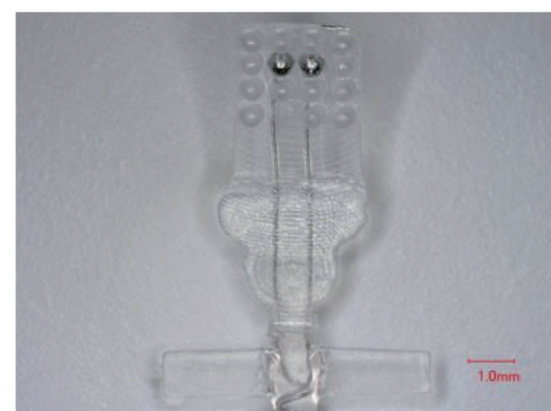

(a)

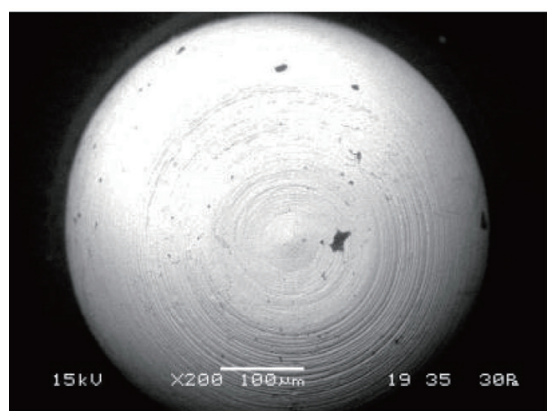

(c)

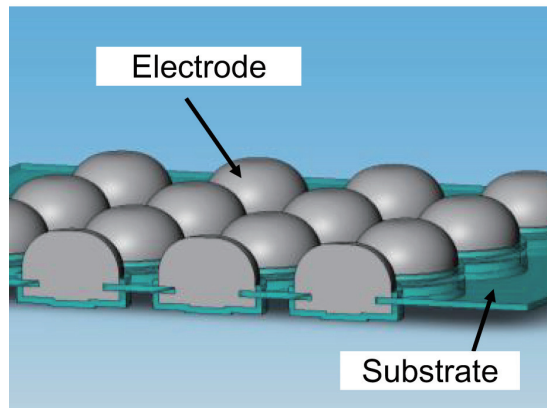

(e)

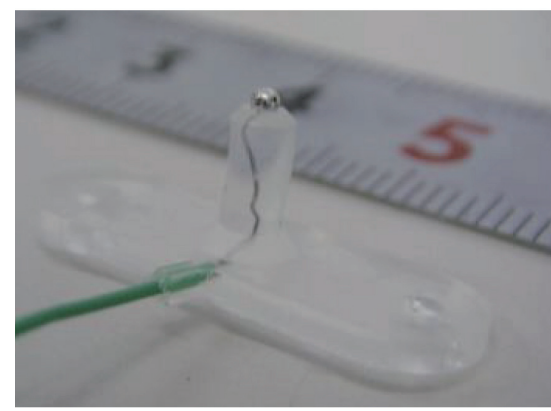

(g)

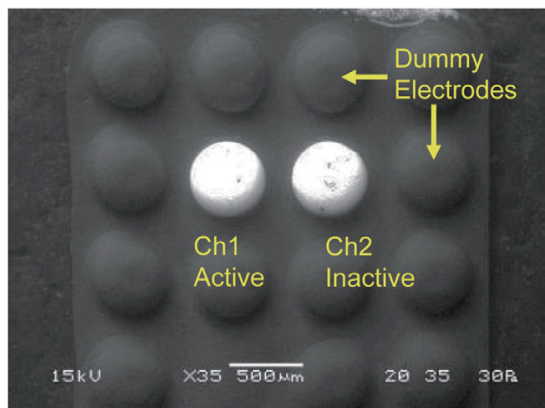

(b)

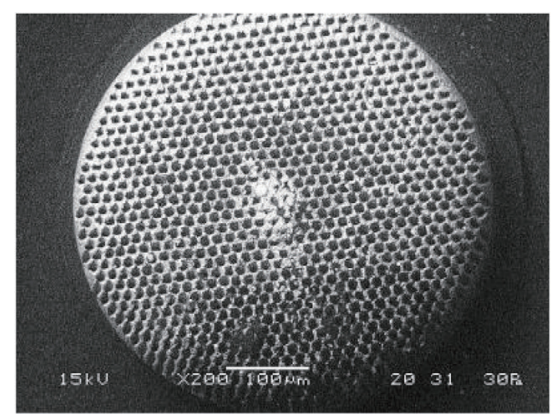

(d)

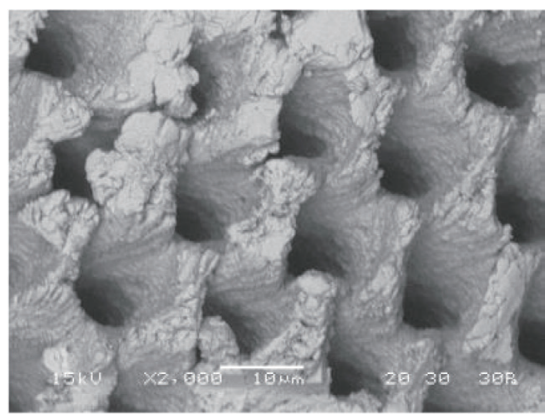

(f)

Fig. 1. (Color online) The two-channel electrode array used in this study. (a) Tip of the array. (b) Magnified view of the array. Electrical stimulation was applied to only one of the two electrodes ("active", channel 1). (c) A smooth-surface electrode. (d) A FLiP electrode. (e) Cross-sectional view of an electrode array. (f) Magnified view of the surface of a FLiP electrode. The typical diameter and depth of a pore are 10 and $100 \mu \mathrm{m}$, respectively. (g) A recording electrode of the type implanted onto the visual cortex. 
$3 \mathrm{~mm}$ from the corneal limbus. A recording electrode [Fig. 1(g)], which is a custom-made 1.4-mm-diameter platinum ball, was placed onto the visual cortex of the rabbit $(8 \mathrm{~mm}$ posterior to the lambda cranial suture and $6.5 \mathrm{~mm}$ lateral to the midline) contralateral to the implanted eye. The recording electrode consisted of a 3-mm-diameter 7-mm-height columnar portion and flat root portion and was made of platinum and polydimethylpolysiloxane [Fig. 1(g)]. A hole of $3 \mathrm{~mm}$ diameter was formed in the skull, and the columnar portion of the recording electrode was inserted into the hole, and the root portion of the electrode was fixed to the skull with 2-mm-diameter stainless steel screws and dental cement (unifast II, GC, Tokyo, Japan). A reference electrode for the measurement of visual evoked potentials (VEPs) or electrical evoked potentials (EEPs), which is a 2-mm-diameter 6-mm-long stainless steel screw, was implanted at the bregma. These electrodes were connected to an intermediate board fixed onto the skull of the rabbit via subcutaneously implanted conductive leads. Details about the experimental system are described in the literature. ${ }^{(1)}$ All in vivo experiments were conducted in accordance with the Association for Research in Vision and Ophthalmology statement on the use of animals in ophthalmic and vision research and with institutional guidelines for the care and use of laboratory animals.

\subsection{Semichronic electrical stimulation}

Two weeks after implantation surgery, an external stimulator was connected to the implanted electrodes. A slip ring was mounted on the roof of the animal cage, and the intermediate box on the skull of the animal and the external stimulator were connected through the slip ring so that the animals could freely move within the cage without breaking the conductive lines with their rotational movement. ${ }^{(11)}$ Charge-balanced cathodic-first rectangular biphasic current pulses with $1.5 \mathrm{~mA}$ amplitude and $0.5 \mathrm{~ms}$ width at a repetition frequency of $50 \mathrm{~Hz}$ were applied to one of the two electrodes [channel 1, Fig. 1(b)] for $8 \mathrm{~h}$ per day for one month. The charge per pulse was $750 \mu \mathrm{C}$. No interphase gap was implemented in the biphasic pulse, i.e., the anodal phase rises up immediately after the end of the cathodal phase.

\subsection{Electrophysiological and electrochemical examinations}

A series of examinations were performed before and after the one-month stimulation. The VEP and EEP were recorded with an amplifier (MEG-6116, Nihon Kohden, Tokyo, Japan). For VEP measurements, after pupil dilation with $0.4 \%$ tropicamide (Mydrin-P, Santen, Osaka, Japan), full-field flash stimulation was applied by a photonic stimulator (SLS-3100, Nihon Kohden) placed $30 \mathrm{~cm}$ from the eye of the rabbit with a setting of $20 \mathrm{~J}$. Evoked potential waveforms were recorded through a 1 to $100 \mathrm{~Hz}$ bandpass filter and were averaged 32 times. For the EEP, cathodic-first 1.0-mA-amplitude 1.0-ms-width current pulses were applied using a custom-made stimulator. Electrical stimulation was repeated 1000 times at $2 \mathrm{~Hz}$, and the averaged waveform was recorded with research software (Eplyzer II, Kissei Comtec, Matsumoto, Japan) after bandpass filtering between $1.5 \mathrm{~Hz}$ and $1 \mathrm{kHz}$. Retinal thickness was evaluated using optical coherence tomography (RS-3000, Nidek, Gamagori, Japan). For the analysis of evoked potentials, the amplitude of VEP was defined as the difference between the baseline and the first negative peak (N1) with a latency of approximately $25 \mathrm{~ms}$. The EEP 
amplitude was defined as the difference between the first positive peak (P1) and the negative peak (N1) with latencies around 10 and $15 \mathrm{~ms}$, respectively.

For impedance measurements, a custom-made platinum reference electrode made of a 0.5 -mm-diameter platinum wire with a surface area greater than $1 \mathrm{~cm}^{2}$ was subcutaneously implanted in the area posterior to the orbit around the temporalis small. Electrode impedance was measured between the stimulation electrode implanted in the sclera and the reference electrode using an electrochemical instrument (PGSTAT12, Metrohm Autolab, Utrecht, Netherlands). A $10-\mathrm{mV}$-amplitude sinusoidal voltage wave was applied for impedance measurements.

\subsection{Charge injection capacity (CIC) in vitro and in vivo}

Before implantation, CICs of stimulation electrodes were measured in $0.01 \mathrm{M}$ phosphatebuffered saline at room temperature. The CIC is defined as the maximum charge density without deviating the water window ( -0.6 to $+0.8 \mathrm{~V}$ vs $\mathrm{Ag} / \mathrm{AgCl}$ for platinum electrodes $)^{(12)}$ during charge injection. Cathodic-first 0.5 -ms-duration current pulses at $30 \mathrm{~Hz}$ were used for measurements. Electrode potential during pulsing was recorded with a custom-made amplifier and oscilloscope (DL750, Yokogawa Meters \& Instruments, Tokyo, Japan). The same stimulation electrode measurement was also performed after explantation. During the stimulation period, the CIC was measured in vivo before and after the one-month stimulation. Detailed measurement procedures are reported elsewhere. ${ }^{(13)}$ Briefly, the $\mathrm{Ag} / \mathrm{AgCl}$ reference electrode was electrically connected to the rabbit via a needle and saline-filled tube. In vivo electrode potential was measured with reference to the reference electrode.

\subsection{Histological analysis}

After finishing the poststimulation examination, the eyes were enucleated and fixed in a mixture of $1.5 \%$ glutaraldehyde and $3 \%$ paraformaldehyde. Following dehydration and embedding in paraffin wax, tissue sections were cut and stained with hematoxylin and eosin.

\subsection{Morphological evaluation of stimulation electrodes}

The surfaces of the stimulation electrodes were examined before implantation and after explantation using scanning electron microscopy (JSM-5600LV, Japan Electron Optics Laboratory, Tokyo, Japan). Prior to the examination of explanted electrodes, electrodes were cleaned using a $0.1 \%$ enzyme solution (Bioplase AL-15-FG, Nagase ChemteX, Osaka, Japan) at $50{ }^{\circ} \mathrm{C}$ for over $3 \mathrm{~h}$ to remove adhered organic material from their surfaces.

\section{Results}

Semichronic STS was applied for four weeks to seven animals. The actual stimulation duration was not the same in all animals (Table 1). This is because sometimes stimulation or recording was not possible owing to mechanical breakage of the experimental system that connected the external stimulator and electrodes. 


\subsection{Safety of semichronic STS}

\subsubsection{Optical coherence tomography}

Visualization of the stimulation electrodes implanted in the sclera with optical coherence tomography was successfully performed in five of seven eyes (Fig. 2). For two eyes, optical coherence tomography imaging was not possible due to corneal opacity that occurred after implantation surgery (Table 1). The smooth-surface electrodes were more clearly visualized than the FLiP electrodes due to the higher reflectance of the smooth-surface electrodes. No abnormality, such as retinal thinning or destruction of retinal layers, was observed. There was no statistically significant difference in retinal thickness before and after the one-month semichronic stimulation for either the smooth-surface or FLiP electrodes (paired $t$-test, Table 2).

Table 1

Summary of the one-month stimulation cohort.

\begin{tabular}{|c|c|c|c|}
\hline Subject ID & $\begin{array}{l}\text { Electrode } \\
\text { surface }\end{array}$ & $\begin{array}{l}\text { Stimulation } \\
\text { duration }(d)\end{array}$ & Notices \\
\hline T041 & Smooth & 28 & \\
\hline T042 & Smooth & 38 & Stimulation duration extended because of system trouble. \\
\hline T043 & Smooth & 40 & Stimulation duration extended because of system trouble. \\
\hline T029 & FLiP & 27 & $\begin{array}{l}\text { Optical coherence tomography (OCT) unavailable because of corneal } \\
\text { opacity. Impedance unavailable because of the failure of reference } \\
\text { electrode. }\end{array}$ \\
\hline T030 & FLiP & 31 & $\begin{array}{l}\text { Impedance unavailable from day } 9 \text { to day } 24 \\
\text { post stimulation because of system trouble. }\end{array}$ \\
\hline T031 & FLiP & 29 & \\
\hline T032 & FLiP & 29 & OCT unavailable because of corneal opacity. \\
\hline
\end{tabular}

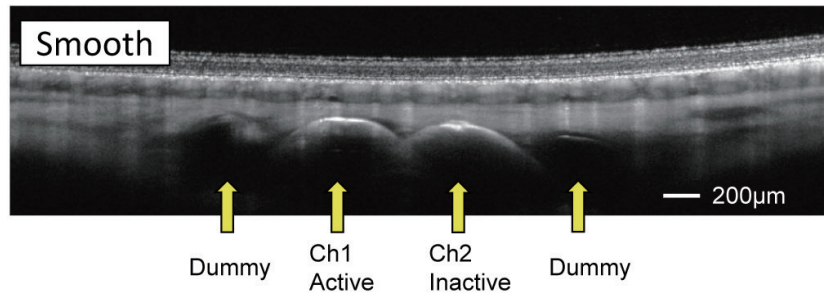

(a)

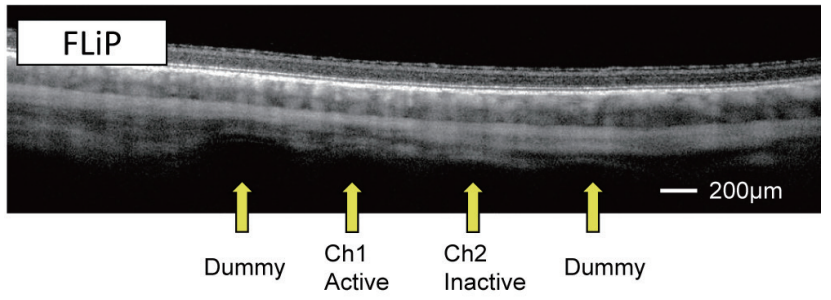

(b)

Fig. 2. (Color online) (a) Visualization of implanted electrodes by optical coherence tomography after one-month stimulation. (b) FLiP electrodes are less visible owing to their lower optical reflectance.

Table 2

Retinal thickness pre- and poststimulation (mean $\pm \mathrm{SD}$ ).

\begin{tabular}{lcl}
\hline & Pre & \multicolumn{1}{c}{ Post } \\
\hline Smooth $(n=3)$ & $149.3 \pm 6.6$ & $144.3 \pm 8.5$ \\
FLiP $(n=2)$ & $149.5 \pm 0.7$ & $144.0 \pm 14.1$ \\
\hline
\end{tabular}




\subsubsection{Histological analysis}

No abnormality, such as in cell number or collapse of the layer structure, was observed in the retina around the active electrode [Figs. 3(c) and 3(d)]. This was the same for both smoothsurface electrodes and FLiP electrodes. The space adjacent to the sidewall of the electrode was filled with connective tissue [Figs. 3(a) and 3(b)]. In contrast, little connective tissue was observed on the top of the electrode, and the scleral tissue was in contact with the tip of the electrode [Figs. 3(e) and 3(f)]. In one eye (Subject ID T032), surrounding tissues could not be observed due to the detachment of the surrounding tissues when removing the electrode from the eyeball during the fixation procedure.

\section{Smooth}

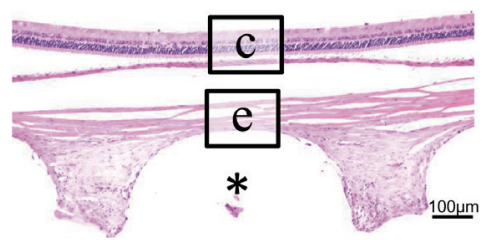

(a)

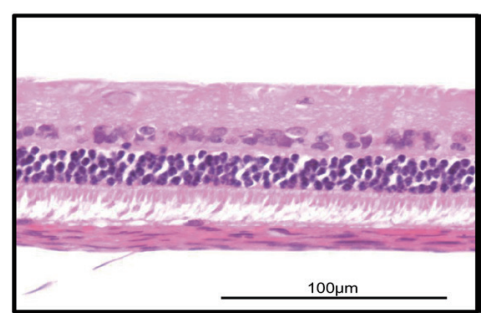

(c)

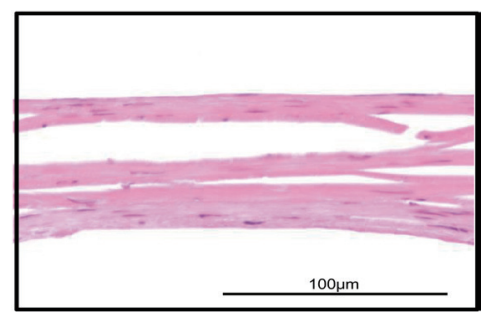

(e)

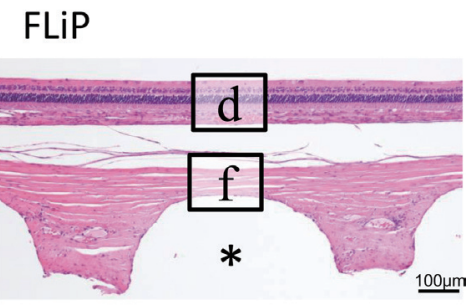

(b)

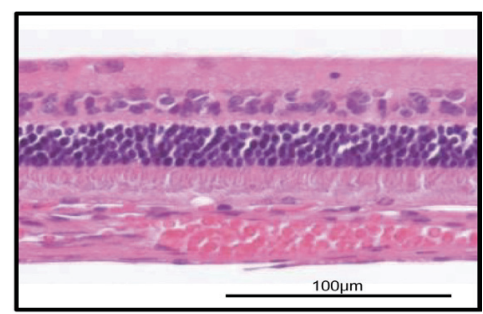

(d)

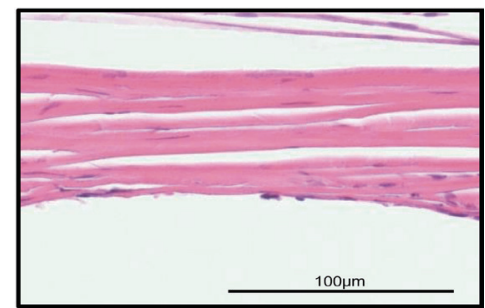

(f)

Fig. 3. (Color online) Cross section of the retina after one-month stimulation. The asterisk represents the position of smooth-surface (a) or FLiP (b) stimulation electrode. Magnified views of the retina adjacent to (c) smooth electrode and (d) FLiP electrode. Magnified views of the sclera adjacent to (e) smooth electrode and (f) FLiP electrode. In (a), the choroid was partially detached due to an artifact during tissue specimen preparation. No abnormality, such as disruption of the retinal layer structure or reduction in cell number, was observed in either retinal tissue. Cell bodies and outer segments of photoreceptors were also well preserved in both tissues adjacent to (c) smooth electrode and (d) FLiP electrode. The space adjacent to the sidewall of the electrode was filled with connective tissue, (a) and (b). In contrast, little connective tissue was observed on the top of the electrode, (e) and (f). 


\subsection{Retinal function after semichronic STS}

VEP and EEP were successfully recorded in all seven animals. Figure 4 shows examples of VEP and VEP waveforms pre- and postsemichronic stimulation. Multiple peaks with similar latency were observed in the waveforms before and after the one-month stimulation. Neither the smooth-surface electrodes nor the FLiP electrodes showed a significant difference in evoked potential amplitudes (VEP and EEP) before and after the one-month stimulation (paired $t$-test).

\subsection{Electrode stability of semichronic STS}

\subsubsection{Longitudinal electrode impedance}

Electrode impedance measured at $1 \mathrm{kHz}$ was approximately constant during the one month of stimulation (Fig. 5). For the active electrodes (i.e., current pulses were applied), their

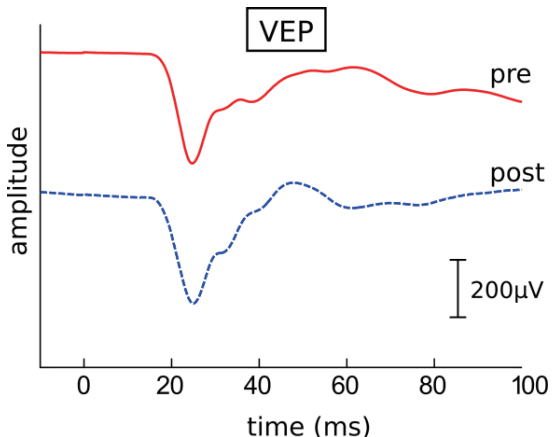

(a)

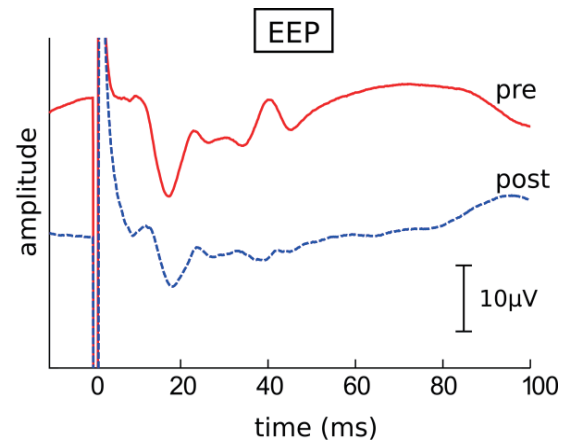

(b)

Fig. 4. (Color online) Examples of (a) VEP and (b) EEP waveforms recorded prestimulation (red) and poststimulation (blue dotted line), respectively.

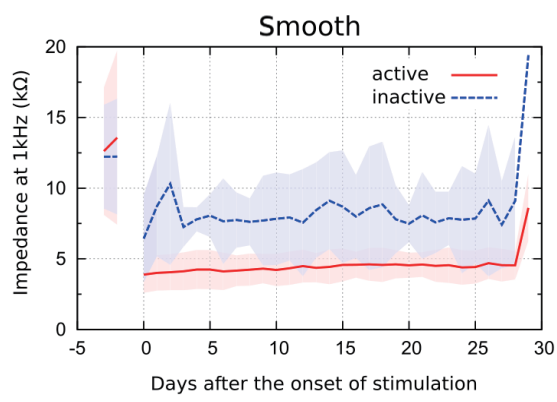

(a)

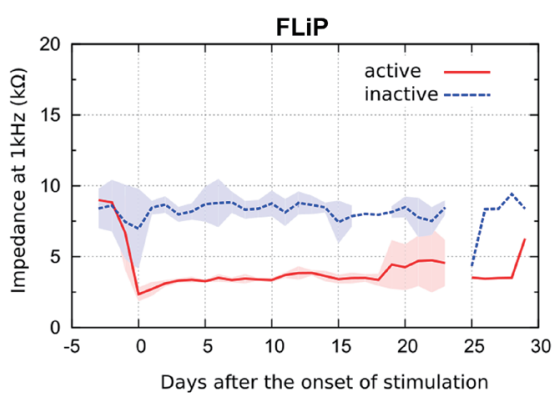

(b)

Fig. 5. (Color online) Longitudinal electrode impedance of smooth [(a), $n=3]$ and FLiP electrodes [(b), $n=3]$. Day 0 represents the initiation of stimulation, and day 28 represents the termination of electrical stimulation. Lines (red, active electrodes; blue dotted, inactive electrodes) and the dilute-colored areas represent means and standard deviations (SD), respectively. In some parts the lines are discontinuous (day -1 for smooth electrode, and day 24 for porous electrode) because data was unavailable because of the problems with the measurement system. The standard deviation of the porous electrodes' active impedance increases markedly from day 19 post-stimulation onset because the impedance of one animal became unavailable because of trouble with the measurement system (T030, Table 1). 
impedance decreased immediately after the initiation of electrical stimulation. Electrode impedance had a tendency to rise again after the termination of stimulation. The impedance of active electrodes was lower than that of the inactive electrodes. In the active electrodes, the FLiP electrodes exhibited lower impedance than the smooth-surface electrodes (red lines in Fig. 5), whereas the inactive electrode showed no clear impedance difference between the smoothsurface and FLiP electrodes (blue lines in Fig. 5).

\subsubsection{CIC}

CICs of the FLiP electrodes were 6-10 times higher than those of the smooth-surface electrodes (Fig. 6). The CICs measured in vivo were approximately $15-20 \%$ of those measured in vitro. There was no significant difference between the active and inactive electrodes. Among the FLiP electrodes, the average in vivo CIC after the one-month stimulation decreased compared with the in vivo CIC prior to the initiation of stimulation, but there was no statistically significant difference. A significant difference was observed only in the smooth-surface electrode between the CIC before and that after implantation measured in vitro.

Table 3

VEP and EEP amplitudes pre- and poststimulation (mean $\pm \mathrm{SD}$ ).

\begin{tabular}{lcccc}
\hline & \multicolumn{2}{c}{$\operatorname{VEP}(\mu \mathrm{V})$} & \multicolumn{2}{c}{ EEP $(\mu \mathrm{V})$} \\
\cline { 2 - 5 } & Pre & Post & Pre & Post \\
\hline Smooth $(n=3)$ & $373 \pm 283$ & $338 \pm 265$ & $6.8 \pm 7.5$ & $15.6 \pm 22.6$ \\
FLiP $(n=4)$ & $512 \pm 181$ & $581 \pm 184$ & $9.9 \pm 5.0$ & $13.6 \pm 6.8$ \\
\hline
\end{tabular}

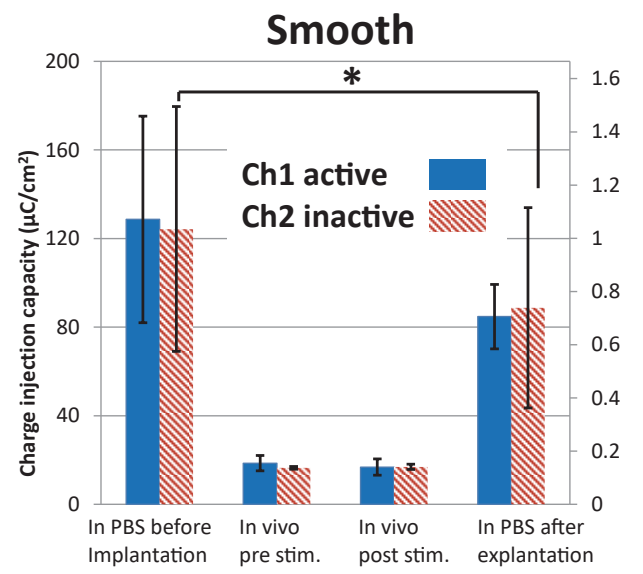

(a)

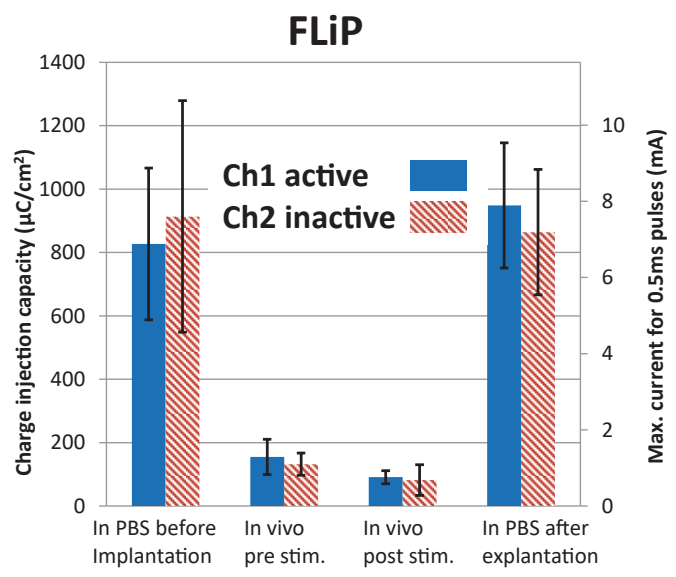

(b)

Fig. 6. (Color online) CICs of (a) smooth-surface electrodes and (b) FLiP electrodes measured before implantation, before one-month stimulation, after one-month stimulation, and after explantation. The solid (blue) and shaded bars (red) correspond to active (current pulses were applied) and inactive electrodes, respectively. The bar length and error bars represent mean and $\mathrm{SD}$, respectively. 


\subsubsection{Electrode surface morphology}

There was no apparent difference in the surface morphology of the electrodes before implantation and after explantation (Fig. 7). This was the same for both smooth-surface electrodes [Figs. 7(a), 7(b), 7(e), and 7(f)] and FLiP electrodes [Figs. 7(c), 7(d), 7(g), and 7(h)].

\section{Discussion}

In optical coherence tomography images that reveal the electrodes in the sclera, no retinal or choroidal bending or thinning induced by mechanical pressure from stimulation electrodes was observed (Fig. 2). In a clinical study conducted with suprachoroidal electrodes, retinal bending was observed around the edge of the electrode array, ${ }^{(6)}$ which was presumably due to mechanical pressure from the array on the retina. The reason why no retinal bending was seen in this study may be because the stimulation electrode was sandwiched inside the sclera, which is a mechanically strong tissue.

There was no significant difference in the thickness of the retina before and after the onemonth stimulation. No abnormality, such as disruption of the layer structure or reduction in the number of cells, was observed in the retina around the stimulation electrode. Similarly, no abnormality was seen in the tissue adjacent to the electrode [sclera and connective tissue; Figs. 3(e) and 3(f)]. Delamination of choroid from photoreceptors was seen in Fig. 3(c), but this was probably due to an artifact of the tissue sample preparation and not to damage of photoreceptor outer segments because of the following two reasons: (1) Delamination occurred not only at the retina adjacent to the electrodes but at other retinal area distant from the electrodes. (2) The delamination was also observed in the histology of the control eye (i.e., the eye without an

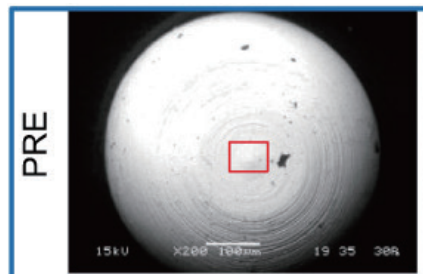

(a)

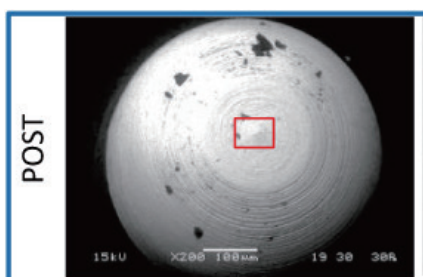

(e)

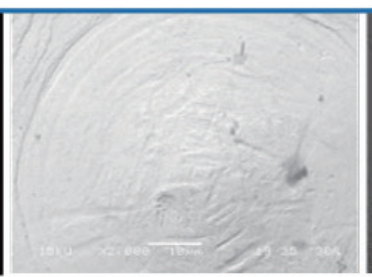

(b)

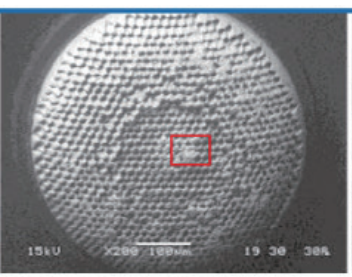

(c)

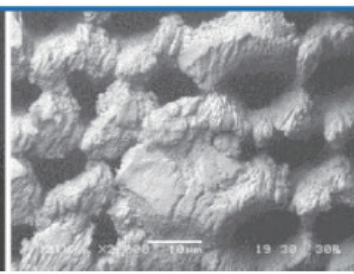

(d)

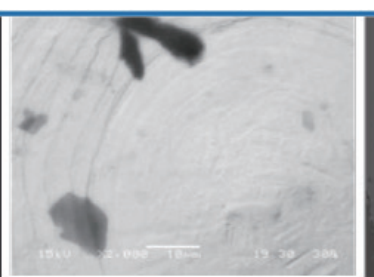

(f)

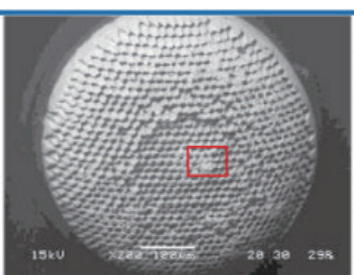

(g)

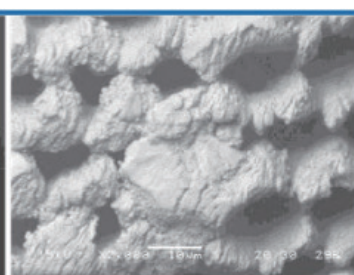

(h)

Fig. 7. (Color online) Electrode surface $(\mathrm{a}-\mathrm{d})$ preimplantation and $(\mathrm{e}-\mathrm{h})$ postexplantation. For both (a, b, e, f) smooth-surface and (c, d, g, h) FLiP electrodes, no apparent morphological change was observed. 
implanted electrode, data not shown). The geometric surface area of the stimulation electrode used in the present study was $0.0043 \mathrm{~cm}^{2}$, which corresponds to a charge density of $175 \mu \mathrm{C} /$ $\mathrm{cm}^{2}$ in the one-month stimulation. However, the actual CIC in vivo was $16-18 \mu \mathrm{C} / \mathrm{cm}^{2}$ for the smooth-surface electrodes and $82-155 \mu \mathrm{C} / \mathrm{cm}^{2}$ for the FLiP electrodes (Fig. 6), indicating that a charge that was 10 times larger than the CIC was continuously injected to the smooth-surface electrodes. Nevertheless, no abnormality was observed in the optical coherence tomography image or histological analysis (Figs. 2 and 3). This suggested that the value of the CIC was very conservative and that the real tissue damage threshold was much higher. However, the voltage waveform recorded in vivo during the one-month stimulation with the smoothsurface electrode exhibited bending in the anodal phase of the biphasic pulse (data not shown), which corresponded to oxygen generation, ${ }^{(14)}$ indicating that an irreversible electrochemical reaction occurred. Nevertheless, no apparent tissue damage was observed. This result presents an interesting question as to whether such a high tissue damage threshold is true for other stimulation sites for retinal prostheses, such as epiretinal, subretinal, and suprachoroidal prostheses. Reports concerning long-term electrical stimulation are quite limited; however, Nayagam et al. implanted 0.6 -mm-diameter platinum electrodes in the suprachoroidal space of cats and applied electrical stimulation for three months and reported that up to $77 \mu \mathrm{C} / \mathrm{cm}^{2}$ chronic electrical stimulation evoked no obvious retinal damage. ${ }^{(7)}$ More recently, Nayagam et al. have reported that localized acute inflammation limited to the suprachoroidal pocket was observed when pulses of $500 \mathrm{nC} /$ phase $\left(63 \mu \mathrm{C} / \mathrm{cm}^{2}\right)$ at 50 pulses/s were applied for four months with 1-mm-diameter platinum electrodes placed in the suprachoroidal space of cats. ${ }^{(15)}$ Additionally, they reported that retinal damage occurred at $580 \mathrm{nC} /$ phase $\left(73 \mu \mathrm{C} / \mathrm{cm}^{2}\right)$ with 100 pulses/s. ${ }^{(15)}$ This suggests that the damage threshold is higher when electrodes are placed inside the sclera than when they are placed in the suprachoroidal pocket. However, because the stimulation frequency, period, and shape of the electrodes were different in our study and these reports, ${ }^{(7,15)}$ further studies are necessary to reach conclusions.

In the present study, a relatively large charge injection of $0.75 \mu \mathrm{C} /$ pulse was applied, but there was no significant difference between the VEP and EEP amplitudes before and after the one-month stimulation, demonstrating the safety and efficacy of the one-month semichronic stimulation from the perspective of retinal function. In STS, a part of the sclera and choroid was between the stimulation electrode and the retina, which was approximately $300 \mu \mathrm{m}$ in rabbits (Fig. 2). Because the retina and the stimulation electrode are $300 \mu \mathrm{m}$ apart, the charge density reaching the retina is reduced because of diffusion. This may explain why no morphological or functional damage to the retina was observed in this study.

Electrode impedance was stable throughout the one-month stimulation period. Furthermore, the impedance of the active electrode decreased immediately after the initiation of stimulation. Although the cause of this is unclear, one possibility is that biomolecules, such as proteins, that were adsorbed to the surfaces of the electrodes were desorbed by the application of the current pulse. Similar phenomena have also been reported in other fields. In a study on cochlear implants, for example, similar trends of longitudinal impedance have been reported. ${ }^{(16)}$ However, in a study on suprachoroidal retinal prostheses, no significant change in impedance was seen before and after the initiation of chronic stimulation. ${ }^{(7)}$ In that study, a small biphasic current pulse (75 $\mu \mathrm{A}$ and $25 \mu \mathrm{s})$ was applied for impedance measurements, and electrode 
impedance was calculated from the voltage waveform, whereas we measured impedance by applying a sinusoidal voltage of $10 \mathrm{mV}$. This may explain the difference in the trend of in vivo impedance. Standard deviations of FLiP electrodes are obviously smaller than those of smooth electrodes both for active electrodes and for inactive electrodes (Fig. 5). Part of this result could be explained by the fact that the impedances of FLiP electrodes were smaller than those of smooth electrodes, therefore the standard deviations of FLiP were also smaller. Another possible explanation is fluctuations in electrode properties in the fabrication process. The electrode impedance had considerable individual variability, possibly owing to the fluctuation of surface area covered by parylene [Fig. 1(e)]. Both smooth electrodes and FLiP electrodes had this variability of surface coverage, but the impedance of FLiP electrodes was less affected by this variability because the porous surface, which was the major factor in electrode impedance of FLiP electrodes, was located at the top of electrodes [Fig. 1(d)] and was less affected by the coverage variability of basal area of electrodes. Actually, the smooth-surface electrode implanted in subject T043 sometimes exhibited large impedance of greater than $10 \mathrm{k} \Omega$, which contributed to the large standard deviations of impedance.

In vivo CIC was approximately $15-20 \%$ compared with that measured in vitro (Fig. 6). The difference between in vitro and in vivo measurements is considered to be due to biomolecule adsorption, tissue encapsulation, and low counterion availability. ${ }^{(12)}$ Similar results have been reported elsewhere. ${ }^{(17-19)}$ The CIC after explantation and washing exhibited a similar value as that measured before implantation (Fig. 6) and no morphological change was seen (Fig. 7), suggesting that electrode deterioration, such as corrosion, did not occur with the one-month electrical stimulation to up to $175 \mu \mathrm{C} / \mathrm{cm}^{2}$. A significant difference in the CIC was observed between the values before implantation and after explantation only in the inactive electrode [channel 2 in Fig. 1(b)] of the smooth-surface electrodes. Although the cause is unclear, one possible explanation is that a small amount of biomolecules remained adhered to the electrode surface even after cleaning due to insufficient washing after extraction, which lowered the CIC. As mentioned above, particularly in smooth-surface electrodes, charge injection much greater than their CIC was performed for one month and an irreversible electrochemical reaction was considered to continuously occur, but no deterioration was observed morphologically (Fig. 7) or electrically (Figs. 5 and 6), suggesting that one-month semichronic electrical stimulation was safe for the stimulation electrode.

In general, retinal prostheses are expected to work for longer than 10 years in the human body, but preclinical testing over longer terms such as 10 years is extremely difficult. In this study we examined the safety of one-month semichronic stimulation, but one month is insufficient. In the retinal prostheses guidance issued by the Food and Drug Administration (FDA), acute tests with 24-h stimulation using the prosthesis electrodes and long-term tests of at least 6 months using fully functional prosthetic devices are recommended. ${ }^{(20)}$ In long-term tests, the device does not have to be activated and in operation for the entire duration of implantation. Although this guidance does not require long-term stimulation, it is essential to estimate the safety of long-term electrical stimulation for retinal prostheses. One method to estimate the safety of long-term electrical stimulation is to extrapolate short-term results by combining results of one-month semichronic tests and chronic tests (such as 6 months). Therefore we are planning chronic tests using similar methods to those described in this work as the next step. 


\section{Conclusions}

No apparent morphological or functional damage to the retina was observed by one-month semichronic electrical stimulation by STS. Our results suggested that a charge injection up to $175 \mu \mathrm{C} / \mathrm{cm}^{2}$ was safe. However, the data in this study are limited to one month; thus, longer term experiments are necessary for the practical use of STS retinal prosthesis. In the future, we will attempt to conduct a longer term chronic stimulation study to confirm the safety and efficacy of STS.

\section{Acknowledgments}

The authors thank Professor Fujikado, Associate Professor Nishida, and Associate Professor Kanda (Osaka University) for their valuable and important advice on animal experiments. The authors thank Mr. Oosawa, Mr. Saitoh, Mr. Shinomiya, Mr. Kuroro, and Mr. Fukasawa (Nidek Co., Ltd.) for the fabrication of the electrodes used in this study.

\section{References}

1 R. W. Rodieck: The First Steps in Seeing 1st ed. (Sinauer Associates, Sunderland, 1998) Chap. 3.

2 A. Santos, M. S. Humayun, E. de Juan, R. J. Greenburg, M. J. Marsh, I. B. Klock, and A. H. Milam: Arch. Ophthalmol. 115 (1997) 511.

3 A. C. Ho, M. S. Humayun, J. D. Dorn, L. da Cruz, G. Dagnelie, J. Handa, P.-O. Barale, J.-A. Sahel, P. E. Stanga, F. Hafezi, A. B. Safran, J. Salzmann, A. Santos, D. Birch, R. Spencer, A. V. Cideciyan, E. de Juan, J. L. Duncan, D. Eliott, A. Fawzi, L. C. Olmos de Koo, G. C. Brown, J. A. Haller, C. D. Regillo, L. V. Del Priore, A. Arditi, D. R. Geruschat, R. J. Greenberg, and Argus II Study Group: Ophthalmology 122 (2015) 1547.

4 K. Stingl, K. U. Bartz-Schmidt, D. Besch, A. Braun, A. Bruckmann, F. Gekeler, U. Greppmaier, S. Hipp, G. Hortdorfer, C. Kernstock, A. Koitschev, A. Kusnyerik, H. Sachs, A. Schatz, K. T. Stingl, T. Peters, B. Wilhelm, and E. Zrenner: Proc. Biol. Sci. 280 (2013) 20130077.

5 H. Sakaguchi, T. Fujikado, X. Fang, H. Kanda, M. Osanai, K. Nakauchi, Y. Ikuno, M. Kamei, T. Yagi, S. Nishimura, M. Ohji, T. Yagi, and Y. Tano: Jpn. J. Ophthalmol. 48 (2004) 256.

6 L. N. Ayton, P. J. Blamey, R. H. Guymer, C. D. Luu, D. A. X. Nayagam, N. C. Sinclair, M. N. Shivdasani, J. Yeoh, M. F. McCombe, R. J. Briggs, N. L. Opie, J. Villalobos, P. N. Dimitrov, M. Varsamidis, M. A. Petoe, C. D. McCarthy, J. G. Walker, N. Barnes, A. N. Burkitt, C. E. Williams, R. K. Shepherd, P. J. Allen, and B. V. A. R. Consortium: PLoS One 9 (2014) el15239.

7 D. A. X. Nayagam, R. A. Williams, P. J. Allen, M. N. Shivdasani, C. D. Luu, C. M. Salinas-LaRosa, S. Finch, L. N. Ayton, A. L. Saunders, M. McPhedran, C. McGowan, J. Villalobos, J. B. Fallon, A. K. Wise, J. Yeoh, J. Xu, H. Feng, R. Millard, M. McWade, P. C. Thien, C. E. Williams, and R. K. Shepherd: PLoS One 9 (2014) e97182.

8 Y. Terasawa, H. Tashiro, Y. Nakano, T. Tokuda, and J. Ohta: Adv. Biomed. Eng. 5 (2016) 137.

9 Y. Terasawa, H. Tashiro, Y. Nakano, K. Osawa, and M. Ozawa: Proc. 2014 Neural Interfaces Conf. (NIC, 2014) 81.

10 Y. Terasawa, H. Tashiro, Y. Nakano, Y. Nakano, and M. Ozawa: Proc. 2013 IEEE Eng. Med. Biol. Soc. (IEEE EMBC, 2013) 3567.

11 H. Tashiro, Y. Terasawa, M. Kuwabara, K. Osawa, T. Tokuda, J. Ohta, and T. Fujikado: Adv. Biomed. Eng. 6 (2017) 8.

12 S. F. Cogan: Annu. Rev. Biomed. Eng. 10 (2008) 275.

13 H. Tashiro, Y. Terasawa, K. Osawa, M. Ozawa, T. Noda, J. Ohta, and T. Fujikado: Invest. Ophthalmol. Vis. Sci. 53 (2012) E-Abstract 5518.

14 S. B. Brummer and M. J. Turner: IEEE Trans. Biomed. Eng. 24 (1977) 440.

15 D. A. Nayagam, P. C. Thien, C. J. Abbott, M. N. Shivdasani, S. B. Epp, J. Villalobos, C. McGowan, R. Williams, C. D. Luu, C. Salinas-LaRosa, J. Yeoh, O. Burns, A. A. Brandli, C. E. Williams, P. J. Allen, and R. K. Shepherd: Invest. Ophthalmol. Vis. Sci. 58 (2017) E-Abstract 4204.

16 F.-G. Zeng, S. J. Rebscher, Q.-J. Fu, H. Chen, X. Sun, L. Yin, L. Ping, H. Feng, S. Yang, S. Gong, B. Yang, H. -Y. Kang, N. Gao, and F. Chi: Hear. Res. 322 (2015) 188. 
17 S. F. Cogan: Proc. 2006 IEEE Eng. Med. Biol. Soc. (IEEE EMBC, 2006) 882.

18 S. R. Kane, S. F. Cogan, J. Ehrlich, T. D. Plante, and D. B. McCreery: Proc. 2011 IEEE Eng. Med. Biol. Soc. (IEEE EMBC, 2011) 5416.

19 Z. Hu, P. R. Troyk, T. P. Brawn, D. Margoliash, and S. F. Cogan: Proc. 2006 IEEE Eng. Med. Biol. Soc. (IEEE EMBC, 2006) 886.

20 U. S. Food and Drug Administration (FDA): homepage of FDA, https:/www.fda.gov/RegulatoryInformation/ Guidances/ucm341954.htm (accessed August 2017).

\section{About the Authors}

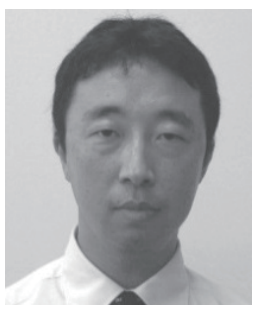

Yasuo Terasawa received his B.S. degree in Applied Physics and his M.S. degree in Information Science from Tohoku University, Miyagi, Japan in 1996 and 1998, respectively. He joined Nomura Research Institute in 1998. Since 2001, he has been a researcher at the Artificial Vision Institute of Nidek Co., Ltd., Aichi, Japan. He received his Ph.D. degree in Materials Science in 2009 from Nara Institute of Science and Technology, Nara, Japan. Since 2009, he has been a research fellow at Nara Institute of Science and Technology and a researcher at Nidek. Since 2016, he has been a manager of the Vision Institute at Nidek. His research interests include electrode technology, microfabrication, neural interfaces, and implantable electronics. He is a member of IEEE Engineering in Medicine and Biology Society and the Association for Research in Vision and Ophthalmology.

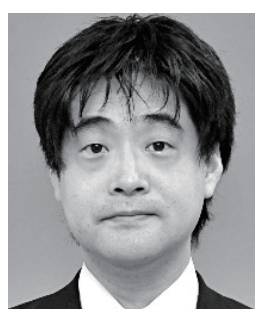

Hiroyuki Tashiro received his B.E. and M.E. degrees in Electrical and Electronic Engineering from Toyohashi University of Technology, Aichi, Japan, in 1994 and 1996, respectively. He received his Ph.D. degree in Engineering from Nara Institute of Science and Technology, Nara, Japan, in 2017. In 1997, he worked as a clinical engineer at Kitasato University Hospital, Kanagawa, Japan. In 1998, he joined Nidek Co., Ltd., Aichi, Japan, where he worked on the research and development of ophthalmic surgical systems. He has been working on the development of retinal prosthesis devices since 2001 . Since 2004, he has been an Assistant Professor in the Faculty of Medical Sciences, Kyushu University, Fukuoka, Japan. His research interests include biocompatibility and physiological evaluation of implantable electrical systems. He is a member of the Japanese Society of Medical and Biological Engineering, Japanese Society for Artificial Organs, the Association for Research in Vision and Ophthalmology, and IEEE Engineering in Medicine and Biology Society.

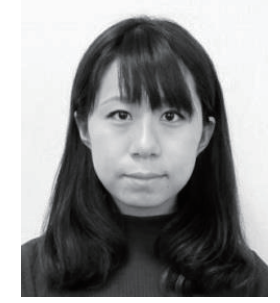

Yukari Nakano received her B.S. degree in Chemical Engineering from Shizuoka University, Shizuoka, Japan in 2009. In 2009, she joined Nidek Co., Ltd., Aichi, Japan. Since 2012, she has been a researcher at the Artificial Vision Institute of Nidek Co., Ltd. Her research interests include the development of active implantable devices for blind people and their electrophysiological evaluation and statistical analysis. She is a member of the Japanese Society of Ophthalmological Optics and the Association for Research in Vision and Ophthalmology. 


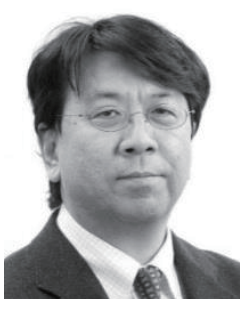

Jun Ohta received his B.E., M.E., and Dr. Eng. degrees in Applied Physics, all from the University of Tokyo, Japan in 1981, 1983, and 1992, respectively. In 1983, he joined Mitsubishi Electric Corporation, Hyogo, Japan. From 1992 to 1993 , he was a visiting scientist at the Optoelectronics Computing Systems Center, University of Colorado at Boulder. In 1998, he joined the Graduate School of Materials Science, Nara Institute of Science and Technology, Nara, Japan as an Associate Professor. He was appointed Professor in 2004. His current research interests include smart CMOS image sensors for biomedical applications and retinal prosthetic devices. He serves as an Associate Editor of IEEE Sensors Journal and as part of the Editorial Board of the Journal of Engineering, IET. He is a Fellow of the Japan Society of Applied Physics and the Institute of Image, Information, and Television Engineers and is a Senior Member of IEEE. 